\title{
The Westerbork Hydrogen Accretion in LOcal GAlaxieS (HALOGAS) survey
}

\section{Survey description and pilot observations (Corrigendum)}

\author{
G. Heald ${ }^{1}$, G. Józsa ${ }^{1}$, P. Serra ${ }^{1}$, L. Zschaechner ${ }^{2}$, R. Rand ${ }^{2}$, F. Fraternali ${ }^{3}$, T. Oosterloo ${ }^{1,4}$, R. Walterbos ${ }^{5}$, \\ E. Jütte ${ }^{6}$, and G. Gentile

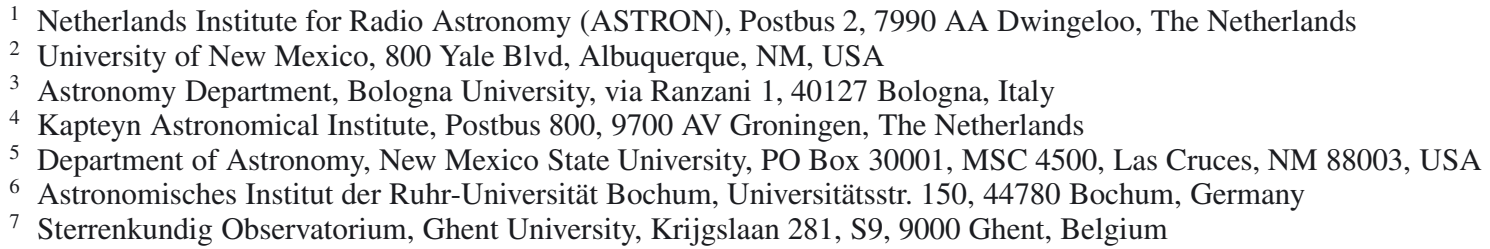

A\&A 526, A118 (2011), DOI: 10.1051/0004-6361/201015938

Key words. galaxies: spiral - galaxies: evolution - galaxies: ISM - galaxies: halos - galaxies: kinematics and dynamics errata, addenda

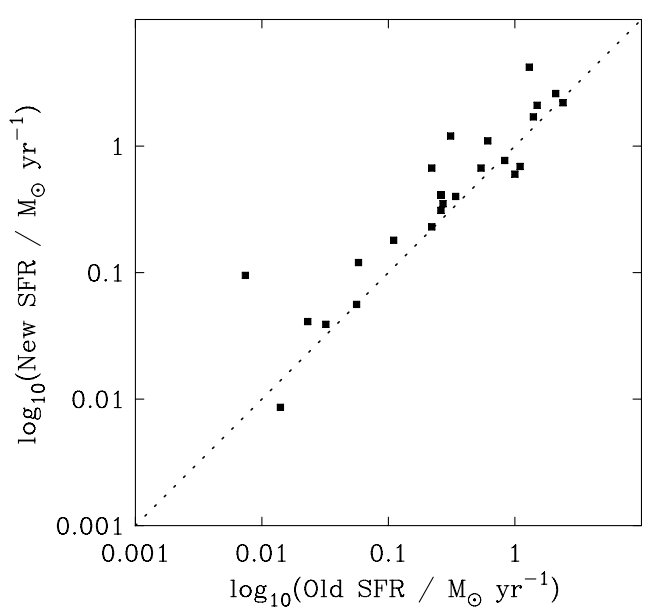

Fig. 1. Comparison between corrected SFR values and the original values. The line represents equal SFR values. Most corrected SFR values are higher, because $d_{\mathrm{T} 88}$ tends to be lower than our adopted distances.

We discovered that in the original paper, the adopted distances $\left(d_{\text {best }}\right)$ were not used in the calculation of the star formation rate (SFR) given in Col. (12) of Table 1. Instead we inadvertently used the Tully (1988) distances $\left(d_{\mathrm{T} 88}\right)$. Here we correct this error, for reference in future HALOGAS publications. The newly computed SFRs are compared to the originally published values in Fig. 1. The results of the original paper are not affected by this correction. Our adopted distances are unchanged from the original paper, but are repeated here for clarity. A description of the origin of the adopted distances can be found in the original paper. A description of the methods used for calculating the SFR values themselves can also be found in the original paper.

\section{References}

Moshir, M., et al. 1990, in IRAS Faint Source Catalogue, version 2.0 Tully, R. B. 1988, Nearby galaxies catalog (Cambridge Univ. Press)

Table 1. Updated SFR values for the HALOGAS sample.

\begin{tabular}{cccccc}
\hline \hline UGC & Other IDs & $\begin{array}{c}d_{\text {T88 }} \\
(\mathrm{Mpc})\end{array}$ & $\begin{array}{c}d_{\text {best }} \\
(\mathrm{Mpc})\end{array}$ & $\begin{array}{c}\text { Old SFR } \\
\left(M_{\odot} \mathrm{yr}^{-1}\right)\end{array}$ & $\begin{array}{c}\text { New SFR } \\
\left(M_{\odot} \mathrm{yr}^{-1}\right)\end{array}$ \\
\hline $\mathbf{1 2 5 6}$ & NGC 0672 & 7.5 & 7.6 & 0.22 & 0.23 \\
1831 & NGC 0891 & 9.6 & 9.2 & 2.4 & 2.2 \\
$\mathbf{1 9 1 3}$ & NGC 0925 & 9.4 & 9.1 & 0.83 & 0.77 \\
1983 & NGC 0949 & 10.3 & 11.3 & 0.26 & 0.31 \\
$\mathbf{2 0 8 2}$ & - & 10.7 & 14.4 & 0.023 & 0.041 \\
2137 & NGC 1003 & 10.7 & 11.6 & 0.34 & 0.40 \\
3918 & NGC 2403 & 4.2 & 3.2 & 1.0 & 0.60 \\
4278 & IC 2233 & 10.6 & 13.6 & 0.11 & 0.18 \\
4284 & NGC 2541 & 10.6 & 12.0 & $<0.27^{a}$ & $<0.35^{a}$ \\
5572 & NGC 3198 & 10.8 & 14.5 & 0.61 & 1.1 \\
7045 & NGC 4062 & 9.7 & 16.9 & 0.22 & 0.67 \\
7322 & NGC 4244 & 3.1 & 4.4 & 0.058 & 0.12 \\
7353 & NGC 4258 & 6.8 & 7.6 & 1.4 & 1.7 \\
& (M 106) & & & & \\
7377 & NGC 4274 & 9.7 & 19.4 & 0.31 & 1.2 \\
7539 & NGC 4414 & 9.7 & 17.8 & 1.3 & 4.2 \\
7591 & NGC 4448 & 9.7 & 9.7 & 0.056 & 0.056 \\
7766 & NGC 4559 & 9.7 & 7.9 & 1.1 & 0.69 \\
$\mathbf{7 7 7 2}$ & NGC 4565 & 9.7 & 10.8 & 0.54 & 0.67 \\
7774 & - & 6.8 & 24.4 & 0.0074 & 0.095 \\
7865 & NGC 4631 & 6.9 & 7.6 & 2.1 & 2.6 \\
8286 & NGC 5023 & 6.0 & 6.6 & 0.032 & 0.039 \\
8334 & NGC 5055 & 7.2 & 8.5 & 1.5 & 2.1 \\
& (M 63) & & & & \\
8550 & NGC 5229 & 6.4 & 5.1 & 0.014 & 0.0086 \\
9179 & NGC 5585 & 7.0 & 8.7 & 0.26 & 0.41 \\
\hline
\end{tabular}

Notes. ${ }^{(a)}$ The SFR value for NGC 2541 is strictly speaking an upper limit, because the IRAS $25 \mu \mathrm{m}$ flux is catalogued as a non-detection by Moshir et al. (1990).

Article published by EDP Sciences 